\title{
COMPARISON BETWEEN THE REACTIVITY OF CARDIOLIPIN ANTIGEN* AND A LECITHIN-FREE CARDIOLIPIN ANTIGEN (CARDCHOL) IN TPI-REACTIVE AND TPI-NON-REACTIVE SERA $\uparrow$
}

\author{
BY \\ HENNING SCHMIDT \\ From the Statens Seruminstitut, Copenhagen, Denmark (Chief: J. Ørskov, M.D.)
}

Following the demonstration by Pangborn (1941) that lecithin and cardiolipin were the active constituents in the "crude" lipoidal antigen employed in the sero-diagnosis of syphilis, Maltaner and Maltaner $(1943,1944,1945)$ and Brown $(1943,1944$ a, b, $1946,1947)$ determined the optimal proportions of these two constituents and of cholesterol for use as antigen in complement-fixation and flocculation reactions for demonstrating reagins.

Maltaner and Maltaner found that the optimal proportions between cardiolipin, lecithin, and cholesterol for a complement-fixation test were $1: 5: 17$, while other ratios were less sensitive.

Brown (1947), examining about 3,900 sera from patients with syphilis, found that the optimal proportions between cardiolipin, lecithin, and cholesterol for a flocculation test were $1: 25: 8$.

Lundbäck (1952) showed that an antigen composed of cardiolipin and cholesterol in the ratio $1: 17$ (i.e. the ratio proposed by Maltaner and Maltaner but omitting lecithin) had the same reactivity with sera from patients with primary syphilis as cardiolipin antigen.

Schmidt (1955a, b, 1956), using a complete cardiolipin antigen and lecithin-free cardiolipin antigen (Cardchol) in complement-fixation tests, examined sera from persons with syphilis and from persons without syphilis who showed biological false positive sero-reactions (B.F.P.). He was able to show a considerable divergence between these two antigens, whose sole difference was the content of lecithin. The B.F.P. sera originated both from patients with no clinical signs or history of syphilis and from patients who became sero-negative on subsequent tests without anti-syphilitic treatment. These sera were not examined for the presence of immobilizing antibodies by means of the TPI test.

\footnotetext{
* Cardiolipin antigen is a mixture of three components (cardiolipin lecithin, and cholesterol) in absolute alcohol.

t Received for publication December 8, 1958.
}

Cardchol, in spite of showing less sensitivity than cardiolipin antigen with sera from syphilitic patients, was nevertheless more non-specific, as it reacted with a greater number of sera from non-syphilitics than did cardiolipin antigen. Several of the B.F.P. sera reacted more strongly with Cardchol than with cardiolipin antigen.

In view of the divergencies in the mode of reaction of the two antigens, an investigation was carried out to determine which serum fractions contained the reagins, and whether there might be several antilipoidal antibodies, each with differing reactivities to cardiolipin antigen and Cardchol. Twelve sera were fractionated by electrophoresis, and the content of reagins in the various fractions was examined, using cardiolipin antigen and Cardchol (Schmidt and Birch-Andersen, 1956). It was shown that those sera, in which the gamma-globulin and gamma plus betaglobulin fractions reacted with Cardchol, also reacted with Cardchol when unfractionated. On the other hand, in certain cases, cardiolipin antigen reacted with the gamma and gamma plus betaglobulin fractions of a serum which, when unfractionated, was non-reactive with cardiolipin antigen. This finding was explained as being due to the presence of an inhibitor which hindered the binding of reagin to cardiolipin antigen. From the varying reactivity of the fractions, the inhibitory action could be ascribed to the albumin plus alpha $1^{-}$ globulin fraction. This was in agreement with the findings of previous authors (Neurath, Volkin, Erickson, Craig, Putnam, and Cooper, 1947; Volkin, 1949). However, sera were also found which, not only unfractionated, but also as far as the different fractions were concerned, reacted with the one antigen but not with the other. This was the case, for example, with a few sera giving a B.F.P. reaction.

The aim of the present communication has been to study the relation between cardiolipin antigen 
and Cardchol in a collection of sera, in most cases reactive, whose content of immobilizing antibodies was known.

\section{Material and Methods}

(a) Antigens.-The following serological methods were employed:

(1) Wassermann - Mørch's complement - fixation test with cardiolipin antigen, C-WR-M (Schmidt, 1951, 1952; Schmidt and Lundbäck, 1954), composed of cardiolipin, lecithin, and cholesterol in the proportions $1: 5: 17$, as proposed by Maltaner and Maltaner (1945).

(2) Cardchol complement-fixation test, using a preparation having the same composition as that for the C-WR-M test, but omitting the lecithin, i.e. cardiolipin and cholesterol in the proportion 1 : 17. About 25 per cent. more complement (fresh guinea-pig serum) is used for the Cardchol test than for the C-WR-M test, as the Cardchol antigen has a slight anti-complementary action (Schmidt, 1955a). The cardiolipin antigen is mixed with 0.9 per cent. saline in the proportion $1: 132$ (the same ratio as for Cardchol) and is then allowed to mature for 20 minutes (Lundbäck, 1952) before the addition of complement to the antigen suspension, whereas complement is added to Cardchol immediately after the antigen has been mixed with saline.

(3) Kahn's standard test, KR (Kahn, 1928), carried out with "crude" antigen.

(4) Meinicke's clarification test, MR (Meinicke, 1932), also carried out with "crude" antigen.

(5) TPI test (Nelson and Mayer, 1949), carried out according to the technique described by Nielsen (1957).

(b) Serological Technique.-The sera were titrated in 0.9 per cent. saline. In the tube with the highest serum concentration there was $0.025 \mathrm{ml}$. undiluted serum, corresponding to a serum concentration of $1 / 13$ after addition of antigen and complement. The proportion between the serum volumes in two consecutive tubes in the titre series is $1: 3$. The haemolysis percentage for the individual tube in the titre series is read by comparison with a haemolysis scale in which the difference between two consecutive tubes is 10 per cent. haemolysis. A logarithmic titre value is then calculated according to Kärber's method (see, e.g. Finney, 1947).

(c) Sera.-All the sera which had been examined in the TPI test on eighteen randomly selected days during the period October, 1957-January, 1958 were examined by the Cardchol test and the C-WR-M test. The content of immobilizing antibodies had thus been determined before the demonstration of reagin (antilipoidal antibody) by cardiolipin antigen and Cardchol. The result of the TPI test was accepted as deciding whether the antılipoidal antibody found was to be taken as indicating syphilis. The TPI test is not a routine procedure, but is carrizd out:
(1) When requested by physicians or hospitals because of presumed disagreement between the serological findings using the lipoidal antigens and the clinical data or history-for example, in cases of suspected syphilis without serological confirmation, or in cases of serological reactivity without corresponding clinical findings or history.

(2) When samples are reactive in two consecutive tests and the accompanying request form does not indicate a history of syphilis.

A positive TPI test was accepted as sufficient basis for the diagnosis of syphilis, while a negative TPI test on a serum reactive with lipoidal antigen was interpreted as indicating that the positive reaction was "non-specific", and could therefore be characterized as a B.F.P. reaction. This basis for classification is not completely correct, as the TPI test can be non-reactive with clinically proven cases of syphilis (e.g. cases of primary syphilis in which the lipoidal antigens give positive results while the TPI test is still non-reactive). As the clinical information shows that the present material contains no sera from patients with fresh syphilis, it has been considered justifiable to employ the TPI test as a criterion for specificity.

\section{Results}

A total of 376 sera were examined, 177 TPIreactive (syphilitic) and 199 TPI-non-reactive (nonsyphilitic). The material was divided into six groups, according to the result obtained in the two complement-fixation reactions C-WR-M and Cardchol (Table I).

TABLE I

TPI-EXAMINED SERA SUBDIVIDED IN SIX GROUPS ACCORDING TO THE RESULTS OF C-WR-M AND CARDCHOL

\begin{tabular}{|c|c|c|}
\hline Group & C-WR-M & Cardchol \\
\hline $\begin{array}{l}1 \\
2 \\
3 \\
4 \mathrm{a} \\
4 \mathrm{~b}\end{array}$ & $\begin{aligned} & 0 \\
& 0 \\
&>0 \\
&>0 \\
&>0\end{aligned}$ & $\begin{array}{c}0 \\
>0 \\
0 \\
0<\text { Cardchol }<\text { C-WR-M }-0.3 \text { log val } \\
\text { C-WR-M }-0.3 \text { log val }<\text { Cardchol }\end{array}$ \\
\hline 5 & $>0$ & Cardchol $>$ C-WR-M \\
\hline
\end{tabular}

Group 4 was split into two sections. In Group $4 b$ the titre with C-WR-M was higher than with Cardchol, but lower than with Cardchol plus 0.3 $\log$ val. The reason for this is that many seropositive cases of syphilis had been observed where Cardchol was reactive, but with a lower titre than with C-WR-M. It was therefore found desirable to carry out a comparison between the two complement-fixation tests at different titre levels.

The Figure (opposite) shows the distribution of C-WR-M and Cardchol for TPI-reactive and TPInon-reactive sera (the number of TPI-non-reactive 


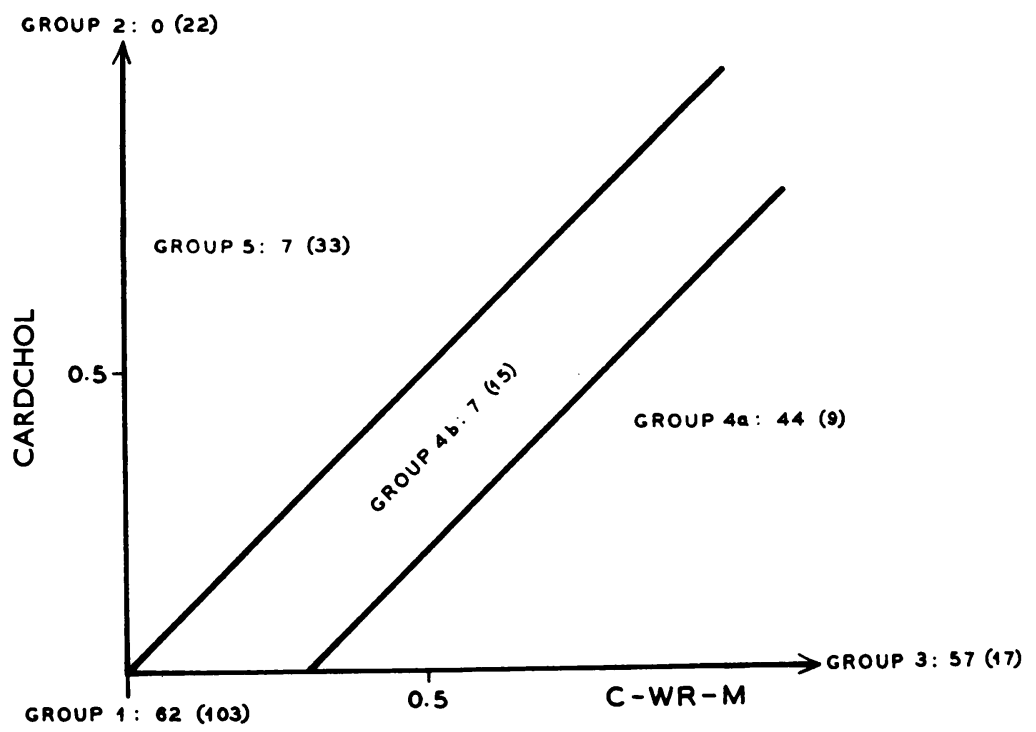

FIGURE.-Distribution of C-WR-M and Cardchol for TPI-reactive and non-reactive sera,

sera in brackets). The TPIreactive sera are mainly represented in Groups 3 and $4 a$, only a few occur in Groups $4 b$ and 5 , and none at all in Group 2.

In Table II the average logarithmic titre values are given for C-WR-M and Cardchol respectively. The titres are given for each of the groups seen in the Figure, excluding Group 1, which consists of sera non-reactive with both C-WR-M and Cardchol. At the same time maximum and minimum titres are given for the two complement-fixation reactions. The distribution of sera in the different groups is determined by the particular nature of the material, i.e. the previously mentioned criteria for

TABLE II

MINIMUM TITRES, MAXIMUM TITRES AND AVERAGE TITRES FOR C-WR-M AND CARDCHOL FOR RESPECTIVELY TPI-REACTIVE AND TPI-NON-REACTIVE SERA BELONGING TO GROUPS 2 TO 5

\begin{tabular}{|c|c|c|c|c|c|c|c|}
\hline \multirow{2}{*}{\multicolumn{2}{|c|}{ Group }} & \multicolumn{3}{|c|}{ C-WR-M } & \multicolumn{3}{|c|}{ Cardchol } \\
\hline & & Min. & $\begin{array}{c}\text { Aver- } \\
\text { age }\end{array}$ & Max. & Min. & $\begin{array}{c}\text { Aver- } \\
\text { age }\end{array}$ & Max. \\
\hline \multirow{2}{*}{2} & TPI - & - & - & - & 0.095 & 0.518 & 1.525 \\
\hline & TPI + & - & - & - & - & - & - \\
\hline \multirow{2}{*}{3} & TPI - & 0.119 & 0.425 & $2 \cdot 7$ & - & - & - \\
\hline & TPI + & 0.048 & 0.538 & $4 \cdot 52$ & - & - & - \\
\hline \multirow{2}{*}{$4 a$} & TPI - & 0.525 & $1 \cdot 153$ & $2 \cdot 15$ & 0.048 & 0.490 & 1.67 \\
\hline & TPI + & 0.930 & $1 \cdot 502$ & $2 \cdot 124$ & 0.477 & 0.981 & $1 \cdot 72$ \\
\hline \multirow{2}{*}{$4 b$} & TPI - & 0.238 & 0.932 & $2 \cdot 6$ & 0.095 & 0.771 & $2 \cdot 395$ \\
\hline & TPI + & $0 \cdot 262$ & 0.859 & 1.74 & 0.072 & 0.722 & $1 \cdot 58$ \\
\hline \multirow{2}{*}{5} & TPI - & 0.048 & 0.571 & 1.65 & 0.191 & 0.8 & $2 \cdot 12$ \\
\hline & TPI + & 0.430 & 0.781 & $1 \cdot 388$ & 0.525 & 0.903 & 1.439 \\
\hline
\end{tabular}

carrying out a TPI test. As the present sera were selected without any consideration for their reaction to Cardchol, it is possible to examine whether the relation between Cardchol and C-WR-M is correlated with the TPI results.

Table III shows the results of this investigation.

Out of 115 sera which were both TPI-reactive and C-WR-M-reactive (Groups 3, 4a, $4 b$ and 5), only seven sera (6 per cent.) reacted more strongly with Cardchol than with C-WR-M, and a further seven sera more strongly with Cardchol than with C-WR-M when 0.3 log val was added to the Cardchol titre (Group 4b). 57 sera (Group 3) (50 per cent.) were reactive with C-WR-M and nonreactive with Cardchol, while none of 62 sera which were TPI-reactive, but C-WR-M-non-reactive, was reactive with Cardchol (Group 1).

74 sera were C-WR-M-reactive and TPI-nonreactive. 33 of these (45 per cent.) were more strongly reactive with Cardchol than with C-WR-M, and 48 (65 per cent.) more strongly reactive with

TABLE III

PERCENTAGE REACTIVITY OF CARDCHOL FOR DIFFERENT COMBINATIONS OF C-WR-M AND TPI IN GROUPS REPRESENTING DIFFERENT RELATIONSHIPS BETWEEN C-WR-M AND CARDCHOL

\begin{tabular}{|c|c|c|c|c|c|}
\hline Group & TPI & C-WR-M & Number & Cardchol > C-WR-M & Cardchol $>$ C-WR-M $-0.3 \log$ val \\
\hline $3,4 a, 4 b, 5 \ldots$ & + & + & 115 & $7(6 \%)$ & $14(12 \%)$ \\
\hline $3,4 a, 4 b, 5 \ldots$ & - & + & 74 & $33(45 \%)$ & $48(65 \%)$ \\
\hline $1 \quad \ldots$ & + & - & 62 & 0 & \\
\hline $1+2 \ldots$ & - & - & 125 & $22(18 \%)$ & \\
\hline
\end{tabular}


Cardchol than with C-WR-M, when $0.3 \mathrm{log}$ val was added to the Cardchol titre.

In the material examined, 6 per cent. (alternatively 12 per cent.) of the TPI-reactive sera are found to be more strongly reactive with Cardchol than with C-WR-M and, by comparison, 45 per cent. (alternatively 65 per cent.) of the TPI-non-reactive sera are found to be more strongly reactive with Cardchol than with C-WR-M.

Tables IV and V show the possible combinations of C-WR-M and TPI, set up as in Table III in relation to the results with KR (Table IV) and MR (Table V).

Table III showed that 6 per cent. of the sera which were reactive with both C-WR-M and TPI were more strongly reactive with Cardchol than with C-WR-M. When the result of the KR test is included in the investigation (Table IV), the material is divided into a further two groups, viz. KR-reactive and KR-non-reactive, and it is then seen that if KR is also reactive, Cardchol is more strongly reactive than C-WR-M in only 3 per cent. of the cases. If, on the other hand, $K R$ is non-reactive, but TPI and C-WR-M are reactive, 36 per cent. of the sera will be more strongly reactive with Cardchol than with C-WR-M. However, the number of sera in the individual groups is small, so the percentage figures calculated must be treated with some reserve. In about 45 per cent. of cases (Table III) Cardchol was more strongly reactive than C-WR-M, when the latter was reactive and TPI was non-reactive. If this group is also sub-divided according to the results with KR, then the number of cases in which Cardchol reacts more strongly than C-WR-M is reduced to 25 per cent. when $\mathrm{KR}$ is reactive; on the other hand, if KR is non-reactive, this group increases to 57 per cent.

If the relationship Cardchol/C-WR-M is investigated for given results of MR and TPI (Table V), it appears that in general the relative reactivities are the same as those demonstrated when $\mathrm{KR}$ was included in the investigation. This must mean that in the present material KR and MR have shown almost the same sensitivity and specificity. Positive reactions in both tests thus lend support to the assumption that the reaction with C-WR-M must be an expression of a syphilitic infection.

As long as the aetiology of the antilipoidal antibodies reactive with Cardchol is unknown, the result with Cardchol is of less interest in sera which are non-reactive with TPI, C-WR-M, KR, and MR.

\section{Discussion}

The lipoidal antigens used in the sero-diagnosis of syphilis are so adjusted (Maltaner and Maltaner, 1945; Brown, 1947; Harris, Rosenberg, and Riedel, 1946; Kline, 1946; and many others) that they are highly sensitive to sera from persons with syphilis, and at the same time as insensitive as possible to sera from persons without syphilis.

However, sera occur which are reactive with one or more of the lipoidal antigens used, although they originate from patients in whom syphilitic infection

TABLE IV

PERCENTAGE REACTIVITY OF CARDCHOL FOR DIFFERENT COMBINATIONS OF KR, C-WR-M, AND TPI

\begin{tabular}{|c|c|c|c|c|c|}
\hline $\mathbf{K R}$ & C-WR-M & TPI & Number & Cardchol > C-WR-M & Cardchol $>$ C-WR-M -0.3 log val \\
\hline $\begin{array}{l}+ \\
+ \\
+ \\
+ \\
- \\
-\end{array}$ & $\begin{array}{l} \pm \\
\pm \\
\pm \\
\pm \\
+\end{array}$ & $\begin{array}{l}+ \\
+ \\
- \\
+ \\
+ \\
+\end{array}$ & $\begin{array}{r}104 \\
14 \\
28 \\
21 \\
11 \\
48 \\
46 \\
104\end{array}$ & $\begin{array}{c}3(3 \%) \\
0 \\
7(25 \%) \\
7(33 \%) \\
4(36 \%) \\
0 \\
26(57 \%) \\
15(14 \%)\end{array}$ & $\begin{array}{c}10(10 \%) \\
16(57 \%) \\
= \\
32 \overline{(70} \%)\end{array}$ \\
\hline
\end{tabular}

TABLE V

PERCENTAGE REACTIVITY OF CARDCHOL FOR DIFFERENT COMBINATIONS OF MR, C-WR-M, AND TPI

\begin{tabular}{|c|c|c|c|c|c|}
\hline MR & C-WR-M & TPI & Number & Cardchol > C-WR-M & Cardchol $>$ C-WR-M -0.3 log val \\
\hline $\begin{array}{l}+ \\
+ \\
+ \\
+ \\
+ \\
=\end{array}$ & $\begin{array}{l}\frac{+}{+} \\
\frac{+}{+} \\
\frac{+}{-}\end{array}$ & $\begin{array}{l}+ \\
\pm \\
\pm \\
+ \\
\pm \\
+\end{array}$ & $\begin{array}{r}106 \\
25 \\
32 \\
13 \\
9 \\
37 \\
42 \\
112\end{array}$ & $\begin{array}{c}5(5 \%) \\
0 \\
10(31 \%) \\
3(23 \%) \\
2(22 \%) \\
00 \\
23(55 \%) \\
22(18 \%)\end{array}$ & $\begin{array}{r}12(11 \%) \\
18 \overline{(56 \%)} \\
2 \overline{(22 \%)} \\
30(\overline{71} \%)\end{array}$ \\
\hline
\end{tabular}


can be excluded. The reason must be that both "crude" antigen and cardiolipin antigen can bind substances in the serum which are not pathognomonic for syphilis. Support for the assumption that the so-called reagins to which the lipoidal antigens become bound are antibodies of antilipoidal nature, is provided by the reacting substances being found in the gamma- and beta-globulin serum fraction, where antibodies usually appear, and by their close connexion with the syphilitic infection. Normally, the antilipoidal antibodies can be demonstrated in serum 4-6 weeks after the infection, and thus at a considerably earlier stage than the immobilizing antibodies, the occurrence of which is considered to be an almost certain proof of a syphilitic infection.

It is incorrect to designate a positive test from a non-syphilitic person as non-specific, as the reaction is an expression of an antigen-antibody binding. This was stressed by Mohr and Moore (1952), among others, who preferred the designation "biologic false positive sero-reaction". However, this expression is not well-chosen either, as the reaction with lipoidal antigen merely indicates the presence of antilipoidal antibodies, presumably originating as a result of infection. When discussing the serological results, therefore, it is preferable to use the terms "reactive" and "non-reactive", and in the case of the reactive sera, to add treponemal or non-treponemal, without further diagnostic comment.

The evaluation of Cardchol antigen carried out here, and the comparison with cardiolipin antigen, have not been based on a desire to establish Cardchol antigen as a more suitable preparation for investigations in syphilis serology than those already in use. On the other hand, it was felt that comparisons between Cardchol and C-WR-M would help in investigating whether the discrepancy existing in many cases between the results of the two tests indicated the presence of different antilipoidal antibodies, in which event the discrepancy might possibly be of diagnostic use. In the present series of tests, it has thus been shown that the reactivity of Cardchol was greater than the reactivity of C-WR-M in about 50 per cent. of those cases in which the TPI test was non-reactive, but only in about 6 per cent. of those in which the TPI test was reactive.

The significance of lecithin for the reactivity of cardiolipin antigen has long been known. The observation was made that a reduction in the lecithin concentration had the effect of making the antigen more "non-specific", i.e. it reacted more frequently with sera from non-syphilitic subjects, while an increase in the lecithin concentration had the result that fewer sera from patients with syphilis were reactive. The explanation may possibly be that the antilipoidal antibodies produced by the syphilitic infection have their reaction optimum at the experimentally determined lecithin concentration, while antilipoidal antibodies of other origin have their reaction optimum at another lecithin concentration.

Ogata (1953) showed that, in flocculation reactions, sera from leprosy patients reacted more strongly with a cardiolipin antigen in which the cardiolipin/lecithin ratio was $1: 1$, than with a cardiolipin antigen in the proportion $1: 10$, which Ogata employs for demonstrating syphilitic antibodies. Ogata's finding was later confirmed by Kent, Otero, and Harrigan (1957), and Kent, Burke, Carroll, Simonton, and Otera (1958), who worked with reduced lecithin concentrations in both complement-fixation and flocculation reactions. Schmidt (1959), examining sera from leprosy patients, found that Cardchol consistently showed considerably higher titres than C-WR-M.

The stability of a cardiolipin antigen is also affected by the lecithin concentration. Lundbäck (1952) showed that the reactivity of lecithin-free cardiolipin antigen decreased with the time which elapsed between mixing the antigen with saline and adding the complement. If the complement was not added until 3 hours after the antigen had been mixed with saline, the reactivity of the lecithin-free cardiolipin antigen in fact disappeared. The nephelometric curve found for Cardchol at various time intervals after mixing antigen and saline also showed the instability of Cardchol (Hartmann and Reyn, 1955).

The reactivity of the Cardchol antigen appears to be greatest when the antilipoidal antibodies are nonsyphilitic in nature. The stronger reactivity of Cardchol in primary syphilis (Lundbäck, 1952), at a stage when the TPI test is still negative and the reactivity of the lecithin-containing antigens still weak, might suggest that the antilipoidal antibodies appearing at an early stage are different in nature from the antilipoidal antibodies which can be demonstrated at a later stage in the disease. The experiments of D'Alessandro, Oddo, Comes, and Dardanoni (1949) with extracts of Reiter treponemes, in which they showed that antibodies against the various constituents of this treponeme developed at different stages of the infection, support the assumption of the presence of a number of different antibodies.

\section{Summary}

177 TPI-reactive sera and 199 TPI-non-reactive sera were examined simultaneously in two complement-fixation tests. The antigens for the complementfixation tests were: 
(1) "Ordinary" cardiolipin antigen mixed in the proportion prescribed by Maltaner and Maltaner (1945).

(2) A lecithin-free cardiolipin antigen, Cardchol, a mixture of cardiolipin and cholesterol in the same proportions as in the ordinary cardiolipin antigen but containing no lecithin.

For TPI-reactive sera the reactivity of the ordinary cardiolipin antigen was superior to that of Cardchol, whereas the reactivity of Cardchol in about 50 per cent. of the TPI-non-reactive sera was superior to that of the ordinary cardiolipin antigen.

The occurrence of various antilipoidal antibodies reacting with different antigens is discussed, and it is stressed that the reactivity of Cardchol is greater when the antilipoidal antibodies are of nonsyphilitic nature.

\section{REFERENCES}

Brown, R. (1943). "Annual Report of the Division of the Laboratories and Research", N.Y. St. Dept. Hlth, Albany, N.Y., p. 16.

(1944a). Ibid., p. 14

-(1944b). J. Bact., 47, 581

1946). J. Immunol., 52, 17; 53, 171.

(1947). Amer. J. Syph., 31, 304.
D'Alessandro, G., Oddo, F., Comes, R., and Dardanoni, L. (1949). Riv. ist. sieroterap. ital. (Sez. II), 24, 134.

Finney, D. J. (1947). "Probit Analysis", p. 39, University Press, Cambridge.

Harris, A., Rosenberg, A. A., and Riedel, L. M. (1946). J. vener. Dis. Inform., 27, 169.

Hartmann, J., and Reyn, A. (1955). Acta. path. microbiol. scand., 36, 129.

Kahn, R. L. (1928). "The Kahn Test. A Practical Guide." Williams and Wilkins, Baltimore; Baillière, Tindall and Cox, London.

Kent, J. F., Otero, A. G., and Harrigan, R. E. (1957). Amer. J. clin. Path., 27, 539.

_, Burke, J. C., Carroll, D. P., Simonton, L. A., and Otera, A. G. (1958). J. chron. Dis., 7,36 .

Kline, B. S. (1946). Amer. J. clin. Path., 16, 68.

Lundbäck, H. (1952). "Studies on the Wassermann Reaction." Thesis, Uppsala.

Multaner, F., and Maltaner, E. (1943). "Annual Report of the Division of the Laboratories and Research", N.Y., St. Dept. Hith, Albany, N.Y., p. 17.

(1944). Ibid., p. 16.

(1945). J. Immunol., 51, 195.

Meinicke, E. (1932). Zbl. Bakt. Abt. 1 (Orig.), 125, 356

Mohr, C. F., and Moore, J. E. (1952). In "Recent Advances in the Studies of Venereal Diseases. A Symposium." Venereal Studies of Venereal Diseases. A Symposium."

Nelson, R. A., and Mayer, M. M. (1949). J. exp. Med., 89, 369.

Nielson, H. A. (1957). Acta path. microbiol. scand., 40, 119.

Neurath, H., Volkin, E., Erickson, J. O., Craig, H. W., Putnam, F. W., and Cooper, G. R. (1947). Amer. J. Syph., 31, 347.

Ogata, T. (1953). "Riassunti delle Comunicazioni. VI Congresso Internazionale di Microbiologia, Roma, 1953."

Pangborn M. C. (1941). Proc. Soc. exp. Biol. (N.Y.), 48, 484.

Schmidt, H. (1951). Brit. J. vener. Dis., 27, 23.

Schmidt, H. (1951). Brit.

(1952). Ibid., 28, 169. microbiol. scand., 36, 141; 36, 147.

(1956). "Cardiolipin Antigen. En oversigt." ; Thesis, Copenhagen.

(1959). Bull. Wld Hlth Org. To be published

- and Birch-Anderson, A. (1956). Acta. path. microbiol. scand., $39,47$.

and Lundbäck, H. (1954). Ibid., 34, 509.

Volkin, E. (1949). J. Immunol., 61, 143. 